\title{
Restaurações indiretas em resina composta: uma alternativa clínica para molares decíduos
}

\section{Indirect restorations in composite resin: a clinical alternative for deciduous molars}

\author{
Gisele Fernandes Dias \\ Universidade Estadual de Ponta Grossa - UEPG - Ponta Grossa - Brasil \\ Centro Universitário de União da Vitória - UNIUV - União da Vitória - Brasil \\ giodonto@hotmail.com \\ Thais Regina Kummer Ferraz \\ Centro Universitário de União da Vitória - UNIUV - União da Vitória - Brasil \\ thaisreginakummer@hotmail.com \\ Luana Salanti \\ Centro Universitário de União da Vitória - UNIUV - União da Vitória - Brasil \\ lusalanti@hotmail.com \\ Ariane de Mello \\ Centro Universitário de União da Vitória - UNIUV - União da Vitória - Brasil \\ arianedemello@yahoo.com.br \\ Fabiana Bucholdz Teixeira Alves \\ Universidade Estadual de Ponta Grossa - UEPG - Ponta Grossa - Brasil \\ fabi.teixeira@uol.com.br
}

\begin{abstract}
Resumo
Novas técnicas restauradoras vêm sendo desenvolvidas com a finalidade de reabilitar o paciente odontopediátrico baseada no aprimoramento dos materiais restauradores. $\mathrm{O}$ presente caso clínico relata a técnica restauradora indireta de incrustação em resina composta para o segundo molar decíduo com ampla destruição coronária. O elemento dentário foi preparado para restauração indireta, moldado com silicona de condensação e após obteve-se o modelo em gesso. A acomodação da resina composta híbrida em camadas seguida de fotopolimerização, foi completa até a obtenção da restauração indireta. A cimentação da incrustação foi realizada com cimento resinoso e feitos os ajustes oclusais necessários seguido de radiografia final. As restaurações adesivas indiretas consistem em alternativa clínica para amplas destruições coronárias, ao proporcionar funcionalidade e prevenção das desarmonias oclusais na dentição decídua. No relato de caso clínico são apresentadas as etapas clínicas e laboratoriais para o sucesso das restaurações indiretas em resina composta.
\end{abstract}

Palavras-chave: odontopediatria, cárie dentária, dente molar, reabilitação bucal, resinas compostas. 


\begin{abstract}
New restorative techniques have been developed with the purpose of rehabilitating the dental child patient based on the improvement of restorative materials. The present case reports the indirect restorative technique of composite resin inlay for the second deciduous molar with extensive coronary destruction. The dental element was prepared for indirect restoration, molded with condensation silicone and after the model was obtained in plaster model. The accommodation of the hybrid composite resin in layers followed by photopolymerization was complete until the indirect restoration was obtained. The cementation of the inlay was performed with resinous cement and the necessary occlusal adjustments were made followed by final radiography. Indirect adhesive restorations consist of a clinical alternative for extensive coronary destruction, providing functionality and prevention of occlusal disharmonies in the deciduous dentition. In the clinical case reports the clinical and laboratory stages for the success of indirect composite resin restorations.
\end{abstract}

Keywords: pediatric dentistry, dental caries, molar, mouth rehabilitation, composite resin

\title{
1. Introdução
}

Os dentes decíduos exercem um importante papel para o correto desempenho da função mastigatória, articulação, fonação, oclusão, estética e são os melhores mantenedores de espaço. A manutenção dos dentes decíduos durante todo seu ciclo biológico, desempenhando seu papel anatômico e funcional é essencial para o desenvolvimento correto da oclusão na dentição permanente (MENEGAZ et al., 2015).

As extensas destruições coronárias representam um desafio diário na prática da clínica odontológica infantil. Durante muito tempo as coroas de aço e o amálgama foram os principais materiais utilizados na restauração de dentes posteriores. Não diferente da odontologia restauradora no paciente adulto, observa-se o aumento da demanda pelo uso de restaurações estéticas.

As restaurações indiretas têm se mostrado como uma boa alternativa clínica para restauração de dentes posteriores. A sua confecção extrabucal, possibilita ao profissional uma melhor visualização dos detalhes, tempo clínico reduzido e diminuição do desconforto do paciente, fatores importantes que devem ser considerados no atendimento odontopediátrico. Restaurações extensas, principalmente em pacientes infantis, são de difícil execução sendo a técnica de restauração indireta uma boa alternativa para esses casos (KOYUTURK et al., 2013). As restaurações em resina composta vêm sendo bem aplicadas na clínica infantil, pois além de estéticas preservam a estrutura dental e seu desgaste por abrasão é semelhante ao desgaste fisiológico do dente decíduo. Também apresentam longevidade satisfatória, pois quando bem empregadas permanecem estéticas e funcionais durante o ciclo biológico e suas propriedades funcionais são importantes para a manutenção ou recuperação das dimensões verticais e mesiodistais (PIVA; RIBEIRO; COELHO DE SOUZA, 2014).

As restaurações diretas em resina composta limitam-se a preparos menores, de fácil acesso, términos cervicais visíveis e sem perda de cúspides. Quanto maior a extensão do preparo, maiores são as dificuldades para restaurar pela técnica direta e maior desgaste superficial da resina ao longo do tempo (HIRATA, 2011).Portanto, o objetivo deste relato de caso foi descrever a técnica restauradora indireta em resina composta para o segundo molar decíduo direito (dente 85), como alternativa de tratamento para as extensas destruições coronárias de molares na dentição decídua. 


\section{Descrição do caso clínico e metodologia de trabalho}

Paciente do sexo feminino, 6 anos, procurou a clínica de odontopediatria do Centro Universitário de União da Vitória - UNIUV para tratamento odontológico acompanhado de seus responsáveis. Segundo a mãe, a queixa principal era de que "os dentes de leite não estavam caindo". Após a assinatura do termo de consentimento livre e esclarecido, foi realizado o preenchimento da ficha clínica. Durante a anamnese observou que a paciente não tinha comprometimento sistêmico, durante o exame clínico foram diagnosticadas múltiplas lesões cariosas sendo os molares decíduos mais acometidos. Após, foi instituído o plano de tratamento baseado nos achados clínicos e radiográficos. O núcleo familiar foi orientado e motivado em relação aos hábitos alimentares e de higiene bucal.

A imagem radiográfica do segundo molar inferior direito (dente 85 ) não mostrou comprometimento pulpar. $O$ dente 85 clinicamente apresentava extensa destruição coronária, com lesão cariosa ativa (ICDAS 6) e radiograficamente não havia comprometimento pulpar (lesão em dentina interna) (Figura 1). O tratamento proposto foi a restauração indireta em resina composta. Na primeira etapa, a dentina infectada foi removida com uso de instrumentos manuais e também brocas de baixa rotação. Em seguida, foi realizado o selamento da cavidade com cimento de ionômero de vidro químico de alta viscosidade (Ketac Molar Easymix®, 3M ESPE, St. Paul, MN, USA) sob isolamento relativo.

A paciente foi anestesiada para realização do preparo para inlay, expulsivo e com ângulos internos arredondados com as pontas diamantadas cônicas de alta rotação. Após o preparo, a moldagem foi feita com moldeira parcial e silicona de condensação Perfil (Coltene/Vigodent, Rio de Janeiro, Brasil) densa e fluída, na técnica de apenas um passo. Os dentes antagonistas foram moldados com alginato tipo II Avagel (Dentsply Sirona, Salzburg, Áustria) e o registro de mordida em cera utilidade (Clássico, São Paulo, Brasil). O dente foi restaurado provisoriamente com cimento temporário de preenchimento Coltosol (Coltene/Vigodent, Rio de Janeiro, Brasil).

A fase laboratorial consistiu na obtenção de modelos de gesso pedra especial tipo IV Durone (Dentsply sirona, Salzburg, Áustria). O isolamento do modelo foi feito com vaselina sólida (Rioquímica, São José do Rio Preto - SP, Brasil). A resina composta nanoparticulada Filtek Z350 XT (3M ESPE, Irvine, CA, EUA), nas cores A1B e B1E, foi inserida em incrementos de até $2 \mathrm{~mm}$ e cada incremento fotopolimerizado durante $40 \mathrm{~s}$, conforme recomendação do fabricante. A escultura dental seguiu os padrões anatômicos do dente 85 feita com espátula Thompson (Quinelato,Rio Claro, Brasil). Ao final, foi feita a polimerização complementar por três minutos em micro-ondas (Figura 2).

A segunda fase clínica iniciou-se com isolamento absoluto da região, remoção da restauração provisória com auxílio de pontas diamantadas de alta rotação e prova da peça (Figura 3). Durante a prova da peça foi observado a cor, adaptação marginal e os detalhes anatômicos. Foi realizada profilaxia no dente 85 e na peça com escova tipo Robinson, pedra pomes e água. Em seguida, foi realizado o condicionamento da peça com ácido fosfórico 37\% Acid Gel (Villevie, Joinville - SC, Brasil) por 30s, lavada abundantemente com água e seca com jato de ar. O remanescente dental (esmalte e dentina) foi condicionado com mesmo ácido por $15 \mathrm{~s}$, lavado por $15 \mathrm{~s}$ e seco com suaves jatos de ar com cuidado para não ressecar a dentina. Após condicionamento ácido, foi aplicado uma camada do adesivo Adper Scotchbond (3M ESPE, Irvine, CA, EUA) na peça, o qual foi fotopolimerizado por 20s conforme recomendação do fabricante (Figura 4). O dente recebeu o mesmo sistema adesivo, porém com adesivo de dois passos, compostos por primer e adesivo separados. Primeiro, foi aplicado o primer à superfície dentária utilizando aplicador descartável (Microbrush/FGM Joinville - SC, Brasil) e em seguida foi aplicado o terceiro passo do sistema, que é o adesivo propriamente dito, espalhado com leve jato de ar e fotopolimerizado por 20s. Com o dente e peça 
preparados, iniciou-se a manipulação do cimento resinoso de presa dual Allcem (FGM, Joinville - SC, Brasil) sobre placa de vidro com espátula metálica. O cimento foi inserido na peça e no preparo, seguido da restauração indireta em posição com compressão sob o dente e extravasamento do agente cimentante. Foi feita uma fotopolimerização inicial de dois segundos apenas para fixar a peça e os excessos foram removidos com sonda exploradora, para seguir com a fotopolimerização durante 40 segundos recomendada pelo fabricante.

Depois de constatado ausência de excessos e falhas na restauração, o isolamento absoluto foi então removido. O ajuste oclusal foi realizado utilizando papel articulador para determinação e posterior desgaste dos contatos oclusais prematuros. O acabamento foi feito com pontas diamantadas, na sequência da mais granulosa para a mais fina. $O$ polimento foi feito com pontas de silicone para resina (Microdont, São Paulo, Brasil). Ao final, foi feito um polimento adicional com escova rotatória Astrobrush (Ivoclair Vivadent, Barueri - SP, Brasil) (Figura 5). Ao término do caso observou-se restabelecimento das funções estética e mastigatória do dente 85 , com aspecto clínico e radiográfico satisfatórios (Figura 6).

Figura 1: Aspecto clínico e radiográfico inicial; dente 85 (segundo molar decíduo inferior direito)

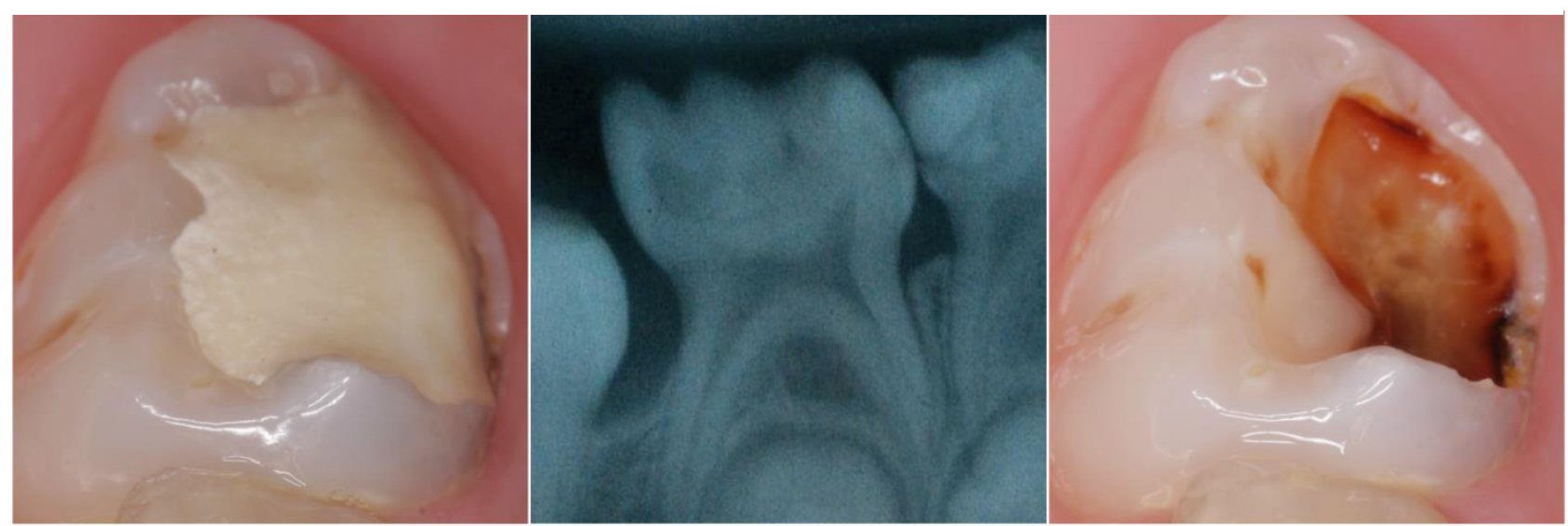

Fonte: Autoria própria (2016)

Figura 2: Moldagem em silicone de condensação; obtenção do modelo em gesso pedra especial e confecção da peça indireta no modelo em gesso

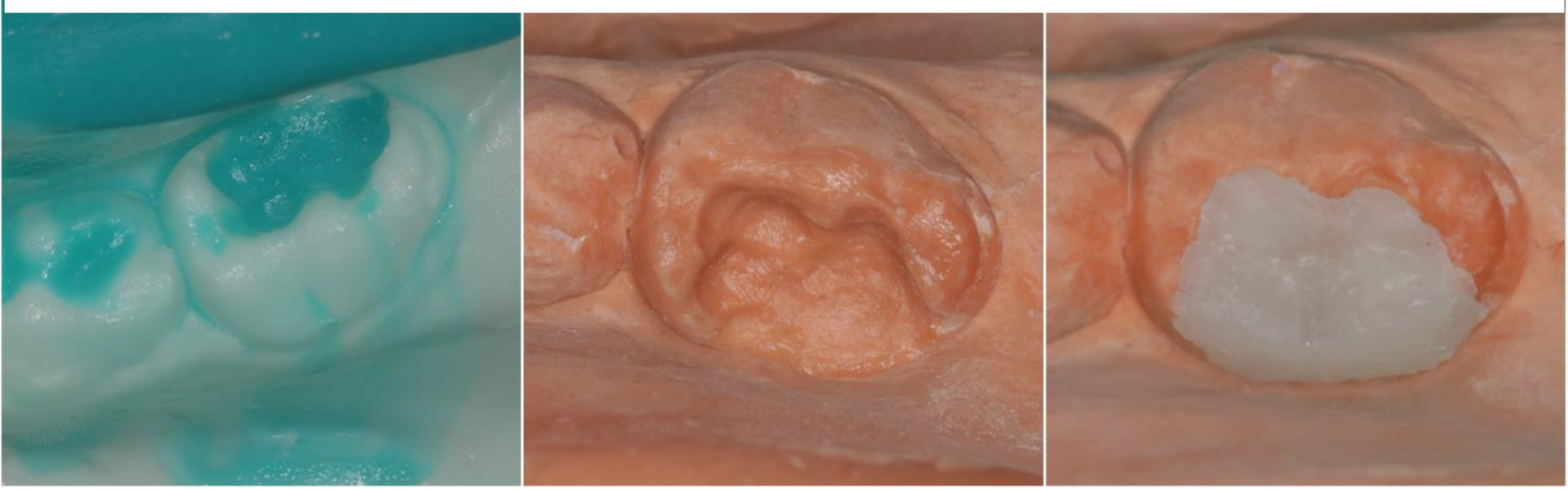

Fonte: Autoria própria (2016)

Figura 3: Prova da restauração indireta 

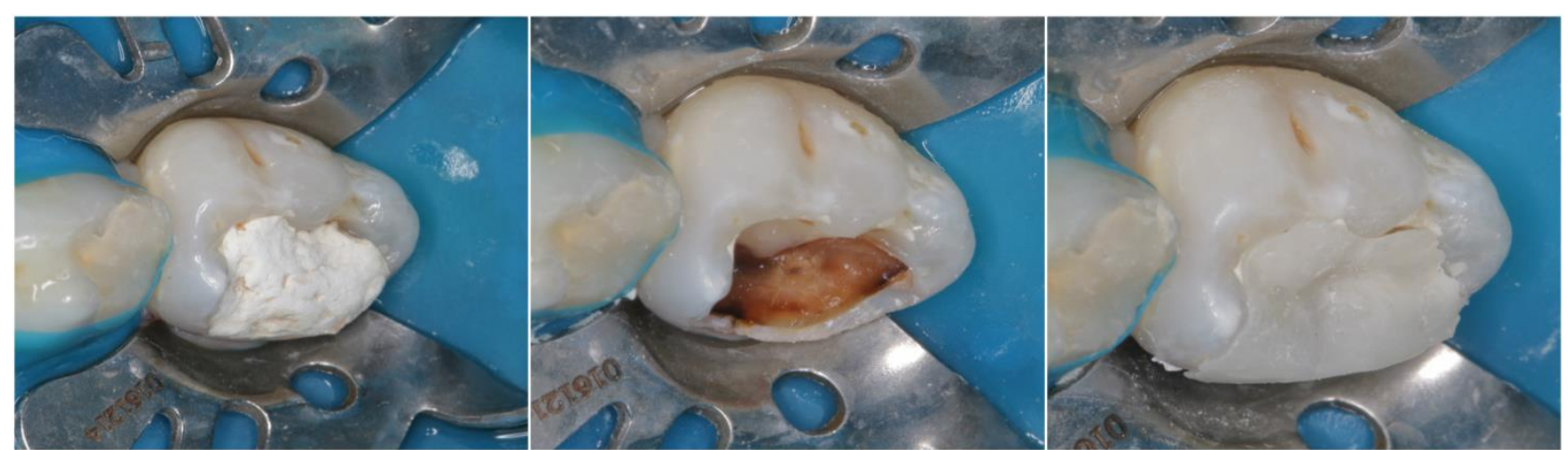

Fonte: Autoria própria (2016)

Figura 4: Procedimento de adesão da restauração indireta para posterior cimentação resinosa.
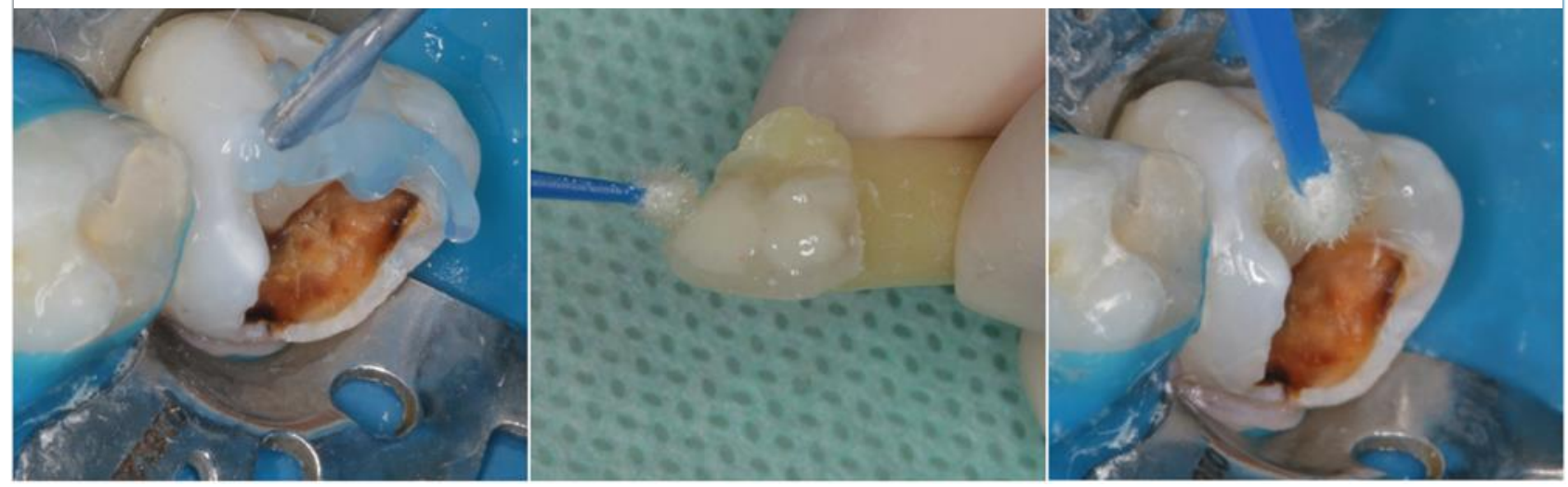

. Fonte: Autoria própria (2016).

Figura 5: Procedimento de acabamento e polimento após a completa cimentação da restauração indireta.
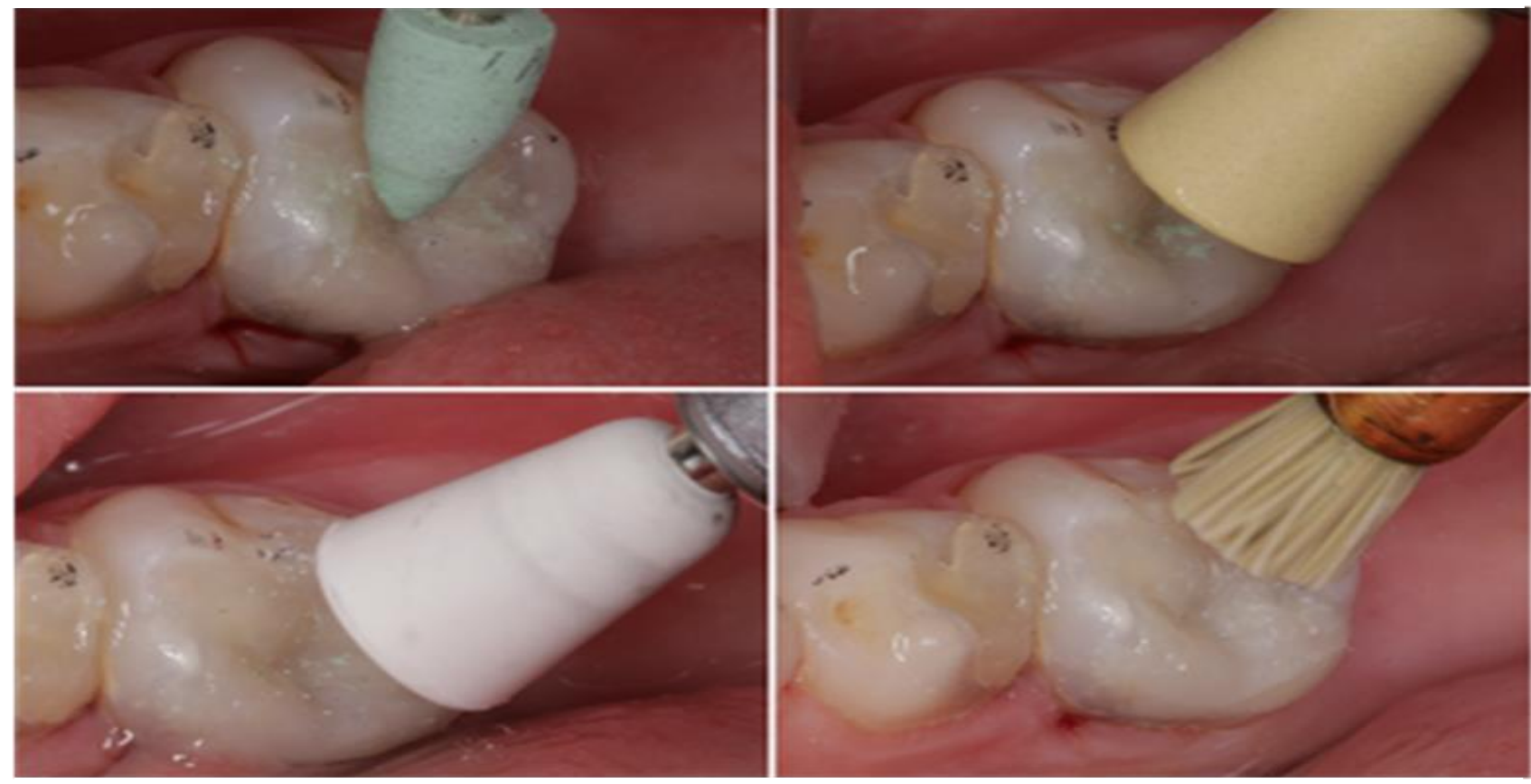

Figura 6: Vista oclusal final da peça cimentada no dente 85; Radiografia final 


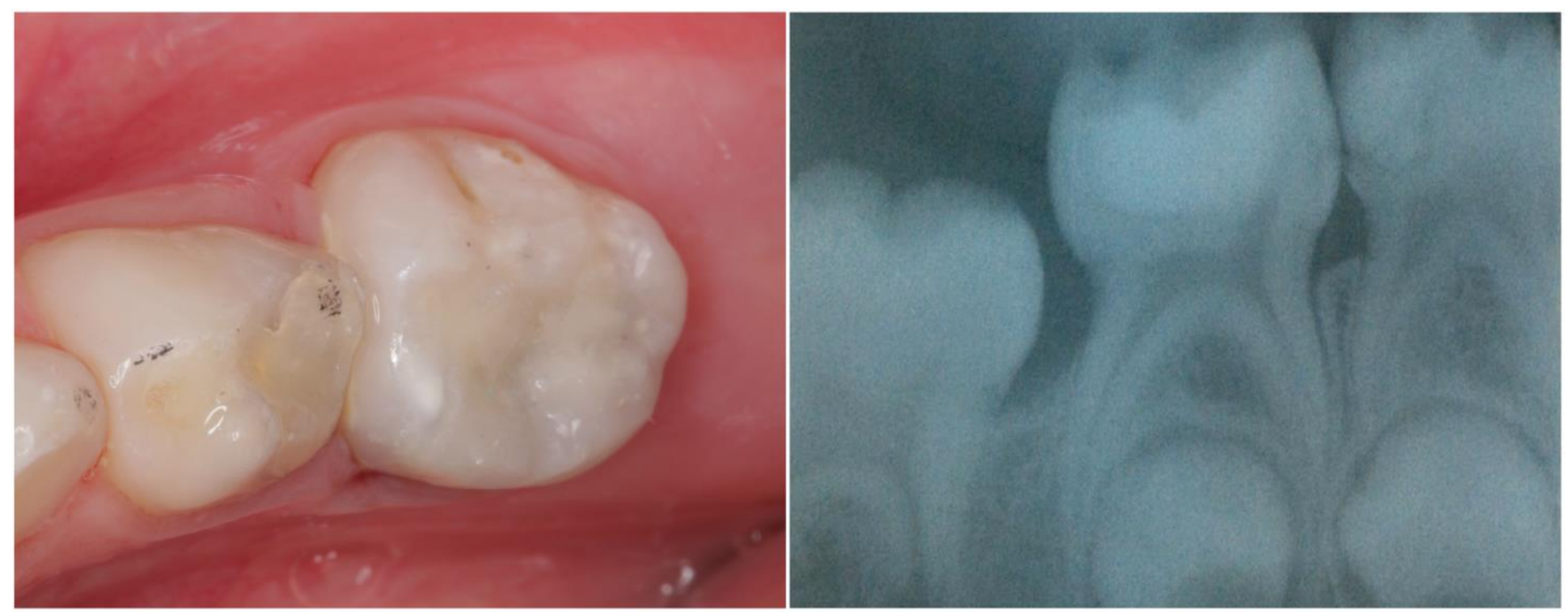

Fonte: Autoria própria (2016).

\section{Resultados e Discussão}

Os procedimentos realizados de forma indireta, como onlays e inlays, são de fato mais caras para o paciente devido à utilização de materiais específicos e custo laboratorial de confecção. Sendo assim, as restaurações feitas em resina composta são uma excelente alternativa para aqueles pacientes que apresentam grandes destruições coronárias em dentes posteriores e precisam de reabilitação, sem um custo elevado (SILVA; MENEZES FILHO; SOUZA, 2007). Quando comparadas às restaurações diretas, estas restaurações permitem uma boa adaptação oclusal e dos contatos proximais, além de redução do efeito negativo de polimerização (CHREPA et al., 2014).

O CIV é muito usado para base e forramento de restaurações em resina composta, pois não provoca danos à polpa dental, age como isolante das alterações térmicas no meio bucal. Esse cimento é indicado em casos onde há dentina esclerótica, pois sobre elas não há uma boa formação da camada híbrida (VIEIRA et al., 2006). O CIV foi mantido como base na restauração como proteção pulpar, pois a liberação de flúor era importante devido à presença de cárie e sensibilidade devido à profundidade da cavidade,

Segundo Lago, Skupien e De Souza (2011), as restaurações feitas de forma indireta apresentam alguns inconvenientes, como a necessidade de duas sessões clínicas e a possibilidade de erros na moldagem e vazamento do gesso, sendo de grande importância que se faça uma boa técnica de moldagem, bem como a correta cimentação. O agente de cimentação desempenha um importante papel na longevidade das restaurações indiretas pois o seu sucesso está relacionado à estabilidade, força e duração da ligação entre o dente e o cimento (MARCONDES et al., 2016). Há controvérsias sobre a remoção do excesso de cimento, que pode acontecer imediatamente após o assentamento da peça, ou após uma polimerização de 3 segundos. A técnica da pré-polimerização facilita a remoção do excesso (CARDOSO et al., 2014). Foi optado pela técnica da remoção após polimerização inicial de 3 segundos, pois assim, o cimento começa a tomar consistência e torna-se mais fácil de remover, sem riscos de deslocamento da peça e também simplifica o acabamento após a cimentação.

Os cimentos de presa dual (Fotoativada e Química) são indicados para a cimentação de restaurações indiretas (GIRALDEZ et al., 2011). A polimerização complementar da resina composta que utiliza forno micro-ondas, proporciona maior grau de conversão de monômeros em polímeros (AROSSI et al., 2007; ARAÚJO; MEDEIROS; RODRIGUES, 2009). Estudos demonstram que as propriedades mecânicas das resinas compostas são melhoradas por meio da polimerização complementar, a qual pode ser 
realizada em equipamentos específicos em laboratórios de prótese e também por equipamentos alternativos, como autoclave, micro-ondas ou estufa de forma aumentar a microdureza da resina previamente fotopolimerizada (ARAÚJO; MEDEIROS; RODRIGUES, 2009).

A utilização de material adesivo e a técnica indireta possibilitou preservação do remanescente dentário (85), reabilitação rápida ao evitar longas sessões de tratamento que interferissem na colaboração da paciente. $O$ objetivo da restauração moderna de dentes decíduos é restaurar a arquitetura estética e funcional da coroa, de acordo com seu valor na oclusão e articulação bem como a influência indireta da restauração sobre o crescimento e o desenvolvimento do sistema estomatognático.

\section{Conclusão}

Os resultados clínicos obtidos após a incrustação mostraram a eficiência da técnica indireta com resina composta, por ser uma alternativa viável, estética, funcional e de baixo custo. As restaurações indiretas inlay/onlay são indicadas para o tratamento de dentes posteriores destruídos por amplas lesões de cárie, pois permite funcionalidade ao reestabelecer a oclusão dentária e permitir a evolução da rizólise. No caso clínico relatado, após exame clí- nico e radiográfico, optou-se pelo tratamento conservador, a fim de evitar as sequelas da extração prematura no desenvolvimento da oclusão aliado aos riscos de inclinação dos dentes adjacentes. Desta forma, foi realizada a reconstrução coronária dos molares decíduos por meio de restaurações adesivas indiretas, para recuperar a anatomia e função aos elementos dentários envolvidos. A principal vantagem do uso da técnica indireta em Odontopediatria é devido a possibilidade de atendimento clínico em sessões curtas comparadas à restauração direta. De forma que, a confecção da restauração é realizada em modelo, sendo que o tempo clínico fica limitado à cimentação e acabamento. Outrossim, não são necessários equipamentos complexos nem encaminhamento ao laboratório, pode ser confeccionada pelo próprio dentista no seu consultório e com os materiais do dia-dia clínico.

\section{Referências}

ARAÚJO, A. R.; MEDEIROS, M. L.; RODRIGUES, C. D. T. Avaliação de três métodos de ativação complementar sobre a dureza superficial de resinas compostas diretas. Revista Odontológica do Brasil Central, Goiania, v. 18, n. 47, p. 28-33, 2009.

AROSSI, G. A. et al. Polimerização complementar em autoclave, micro-ondas e estufa de um compósito restaurador direto. Revista Odontológica Ciência, Porto Alegre, v. 22, n. 56, p. 177-180, abr./jun. 2007.

CARDOSO, P. et al. Facetas cerâmicas: como remover os excessos do cimento resinoso? Clínical International Journal Brazilian Dentistry, v. 10, n. 2, p. 214-225, abr./jun. 2014.

CHREPA, V. et al. The survival of indirect composit resin onlays for the restoration of root filled teeth:a retrospective medium-term study. Journal Endodontic international, v. 47, n.10, p. 967-973, oct. 2014.

GIRALDEZ, I. et al. Early handness of self-adhesive resin cements cured under indirect resin composite restaurations. Journal of Esthetic and Restaurative Dentistry, v. 23, n. 2, p. 116-124, apr. 2011. 
HIRATA, R. Restaurações estéticas e escultura posterior: quando utilizar resinas compostas diretas? In: HIRATA, R. (Org.). Tips: dicas em odontologia estética. São Paulo: Artes Médicas Ltda. 2011. p. 388-389.

KOYUTURK, A. E. et al. Two-vear follow-up of indirect posterior composite restorations of permanent teeth with excessive material loss in pediatric patients: a clinical study. Journal of Adhesive Dentistry, v. 15, n. 6, p. 583-90, Dec. 2013.

LAGO, M.; SKUPIEN, J. A. I.; DE SOUZA, N. C. Restauracões indiretas em resina composta-desmistificação da técnica. International Journal of Dentistry, Recife, v. 10, n. 4, p. 282-286, out./dez. 2011.

MARCONDES, M. et al. Clinical Evaluation of Indirect Composite Resin Restorations Cemented with Different Resin Cements. Journal of Adhesive Dentistry, v. 18, n.1, p. 59-67, jan. 2016.

MENEGAZ, A. M. et al. Efetividade de mantedores de espaço em odontopediatria: revisão sistemática. Revista Faculdade de Odontologia - UPF, Passo Fundo, v. 20, n. 2, p.25257, mai./ago. 2015.

PIVA, F.; RIBEIRO, C. S.; COELHO-DE-SOUZA, F. H. Avaliação clínica de restauracões de resina composta em molares decíduos: estudo piloto. Revista Associação Paulista de Cirurgiões-Dentistas, São Paulo, v. 68, n. 1, p. 69-74, dez. 2014.

SILVA, C. H. V.; MENEZES FILHO, P. F.; SOUZA, F. B. Restauração indireta em resina composta - Uso do calor na polimerização adicional. Odontologia Clínica-científica, Recife, v. 6, n. 1, p. 85-89, jan. 2007.

VIEIRA, I. M. et al. O cimento de ionômero de vidro na odontologia. Revista Saúde.com, Itapetinga, v. 2, n. 1, p. 75-84, abr./jun. 2006. 